\title{
Sensory-Somatic Nervous System
}

National Cancer Institute

\section{Source}

National Cancer Institute. Sensory-Somatic Nervous System. NCI Thesaurus. Code C33533.

The division of the peripheral nervous system that comprises the afferent loop of the somatic nervous system, which functions to relay somatosensory perception, including touch, heat, and cold to the spinal cord and CNS. 